\title{
Las vías anselmiana y ockhamista para razonar a Dios desde sí mismo*
}

\author{
Jorge Francisco Aguirre Sala* \\ Universidad de Monterrey, México
}

Recibido: 26 de agosto de 2009 • Aprobado: 16 de septiembre de 2009

\section{esumen}

Aunque con distintas ontologías, lógicas, teorías lingüísticas (particularmente el uso de la suppositio) y diferencias radicales ante el problema de los universales, Anselmo y Ockham se presentan como dos pensadores medievales que utilizan su razón para auxiliar la fe, y por ello coinciden en la intuición del Fundamento común. La argumentación anselmiana utiliza la suposición semántica, la consecuencia lógica y la implicación material, para "pensar la grandeza de lo mayor" y lanzar al espíritu hacia "lo mayor" que, por definición, apunta más allá, justo en Aquel que el corazón ya cree, utilizando semánticamente la appelatio. Para Ockham, dicha comprehensión no demuestra el predicado "existencia": al distinguir entre "demostración"y "prueba", niega cualquier demostración de la existencia de Dios y considera que las vías del movimiento, la causalidad eficiente y final, son improcedentes porque carecen de evidencia y proceden de la fe. Entonces recurre a la prueba de la suposición lingüística personal, pues "Dios" no le resulta signo intencional meta-lingüístico.

Palabras clave: suposición lingüística, implicación material, denotación, comprehensión, prueba de Dios, existencia del Conservador.

*ste texto es la síntesis de una propuesta de investigación que el autor viene desarrollando en el contexto de los intereses académicos en la Universidad de Monterrey (México).

** Profesor investigador de la Universidad de Monterrey (México). Correo electrónico: joaguirre@udem.edu.mx 


\section{Anselm and Ockham routes to reason God himself}

\section{Abstract}

Although with different ontologies, logical, linguistic theories (particularly the use of suppositio) and radical differences to the problem of universals, Anselm and Ockham are presented as two medieval thinkers who use their reason to assist the faith, and therefore agree the intuition of the common basis. Anselm's argument uses the assumption semantics, the logical and material implication, to 'think the greatness of the greatest' and release the spirit to 'the greatest' which by definition points beyond, right in the heart and one who create, using the appelatio semantically. For Ockham, this comprehension does not prove the predicate "existence" to distinguish between "show" and "test" denies any proof of the existence of God and believes that the routes of movement, efficient and final causation, are irrelevant because they lack evidence and from faith. He then turns to test the assumption of personal language, for "God" is not intentional meta-linguistic sign.

Key words: Assumption linguistic material implication, denotation, comprehension, proof of God's existence Conservative. 


\section{Les voies anselmienne et ockhamiste pour raisoner Dieu à partir de soi}

\section{Résumé}

Malgré les ontologies distinctes, logiques, théories linguistiques (particulièrement I 'emploi de la suppositio) et les différences radicales face au problème des universels, Anselmo et Ockhman se présentent comme des penseurs médiévaux qui utilisent leur raison pour aider la foi, et pour cela, ils coincident dans l'intuition du fondement commun. L'argumentation anselmienne utilise la supposition sémantique, la conséquence logique et l'implication matérielle, pour penser la grandeur de ce qui es superieur et lancer l'esprit vers "le grand" qui, par définition, vise au delà du juste de ce que le coeur croit en utilisant sémantiquement la appelatio.

D'après Ockham, cette compréhension nous fait la démonstration de l'existence du prédicat "existence": en faisant la distinction entre démonstration et vérification, il nie toute manisfestation de l'existence de Dieu et considère que les voies telles que le mouvement, la causalité éfficace et finale sont inoportunes ou irrecevable parce qu'elles manquent d'évidence et proviennent de la foi.

Donc il prend recours à la vérification de la supposition linguïstique personnelle, car "Dieu" ne lui signifie un signe intentionnel meta- linguïstique.

Mots clés: Supposition linguïtique, implication matérielle, témoignage, compréhension, vérification de l'existence de Dieu, exitence du conservateur. 


\section{ntroducción}

¿Cómo razonar la fe desde sí misma?, ¿hay necesidad de ello?, ¿qué caso tiene indagar la verdad si ya creo?, ¿sé cabalmente lo que de suyo creo? Estas preguntas revelan un trasfondo epistemológico para la filosofía y la teología y también ponen en debate los vínculos de la fe y la razón. Muchos consideran que el fideísmo se adelanta al intelecto en su obrar natural. Los dogmas tienen la gran virtud de preceder a la lógica y, en consecuencia, no se requiere pensar. De manera más vulgar, muchas personas creen que ya está escrita la narrativa de las acciones humanas por Dios o el destino. No es el determinismo o la predestinación lo que abordaremos, pero son otro ángulo de la presuposición jerárquica entre fe y razón, donde la razón es utilizada para fundamentar y demostrar la fe y no para dar cuenta de ella y dotar de un mayor y mejor saber sobre lo que ya se cree. El razonamiento es sometido ancilla teología, en vez de utilizarlo para la proyección del sabio contenido de verdad de lo que se cree. Tampoco es el problema moral-epistemológico el que nos ocupará, sino que, tomando en cuenta el indiscutible punto de partida del creyente (Dios existe), nos ocuparemos de dos casos privilegiados que muestran cómo razonar es fe desde sí misma. Abordaremos las extraordinarias experiencias de Anselmo y Ockham. Estos dos filósofos medievales son tan disímiles en sus procedimientos lógicos, sus ontologías y las concepciones acerca de los universales que, a fuerza de responder nuestras preguntas iniciales, nos obsequiaran un hermoso corolario: Si Dios existe, cualesquiera semánticas nos están permitidas.

La cooperación de la razón para obtener más saber de aquello que ya creemos se expresa ejemplarmente en el pensamiento de Anselmo de Canterbury. Este autor pretende "elevar las almas a la contemplación de Dios, se esfuerza en comprender lo que cree" (según él mismo relata en el proemio del Proslogium). Así, la actitud de quien ocupara el arzobispado de Canterbury queda expuesta por la designación original de los primeros títulos a sus opúsculos: "Ejemplo de meditación sobre el fundamento racional de la fe" para el Monologium; mientras que para el Proslogium eligió el título absolutamente revelador de la intención que hemos señalado: "La fe buscando apoyarse en la razón". 
Anselmo da por supuesta la verdad de la fe y utiliza la cooperación de la razón; por ello, no busca el complemento de la razón. La verdad ya ha sido alcanzada. Consecuentemente, una mejor traducción del título del Proslogium sería "La fe pide ser comprendida por la razón", ya que el saber revelado y sobrenatural se posee con anterioridad. Para él como para sus 'hermanos" (quienes le exhortan a redactar los opúsculos'), su corazón ya cree y ama, y así la racionalización discursiva de tales dogmas-verdades le es de utilidad para defenderse de los simples, los necios y los insensatos que han negado a Dios. Pero como la fe es débil ${ }^{2}$, la razón resulta un apoyo para el creyente: ésta explica lo que no puede argumentar aquella, pero sí revela. De tal modo, la razón con la fe es mejor que la fe sin la razón. En este sentido, sabemos que la lógica semiótica auxilia a la fe, de manera similar que lo hace la teología, pues ésta le ayuda a defenderse y a exponer en las implicaciones y consecuencias que conlleva creer. Es muy ilustrativo el siguiente pasaje del Proslogium: "... no intento, Señor, penetrar tu profundidad, porque de ninguna manera puedo comparar con ella mi inteligencia; pero deseo comprender tu verdad que mi corazón cree y ama" (Anselmo, 1952, p. 366).

Anselmo se esfuerza en comprender lo que de antemano cree, no sólo para resistir al insensato, sino para evitar la negligencia, pues la filosofía apuntala con razones los contenidos dogmáticos. Por tanto, Anselmo no defiende el contenido de su fe (fe "objective"), sino el argumento teológico y racional que a su vez protege al contenido de lo que se cree. Las razones necesarias que auxilian la protección no convierten a nadie hacia la fe, sino que la suponen e iluminan. Sin embargo, no es la fidelidad una condición para entender, porque se busca la aportación de la razón especulativa ahí donde la fe no aporta argumentos.

1 “(...) quatenus auctoritate scripturae penitus nihil in ea persuaderetur, sed quidquid per singulas investigationes finis assereret, id ita esse plano stilo et vulgaribus argumentis simplicique disputatione et rationis necessitas breviter cogeret et veritatis claritas patentes ostenderet". En su traducción: "(...) pidiéndome que no me apoyase en la autoridad de las Sagradas Escrituras y que expusiera, por medio de un estilo claro y argumentos... que fuese fiel... a las reglas de una discusión simple y que no buscase otra prueba que la que resalta espontáneamente del encadenamiento necesario de los procedimientos de la razón y de la evidencia de la verdad" (Prologus, Monologium, p. 190).

2 Cfr. La estipulación de la fe en Carta a los Hebreos II; I: la fe carece de evidencia y, por tanto, posee una naturaleza negativa. 
Encontramos así una instrumentación "lógica" al servicio de la creencia, como lo sugiere Henry (1967), pues como Copleston (1979, p. 162) comenta: "la aplicación de la dialéctica a los datos de la teología sigue siendo teología". Advirtamos que Anselmo no desarrolló un tratado de lógica (aunque su obra De Grammatico, se ubica en el campo de la semiótica, como veremos adelante), pero queremos analizar las argumentaciones que utiliza paralelamente a la teología. Sospechamos que en el arte de razonar fue instruido por los tratados de Boecio, Casiodoro e Isidoro, comunes en la educación durante la primera parte de la Edad Media. No cabe duda de su conocimiento de Aristóteles, sobre todo de las Categorías y el Peri Hermeneias, como también de la gramática de Prisciano.

Respecto a las pruebas de índole teológica que elegimos para mostrar su lógica subyacente, la profesora Cabrera Villoro (1985, p. 369) advierte la división usual de los argumentos en dos grupos: "aquellos que parten de la existencia de un mundo ordenado y aquellos que parten de la riqueza de la idea de Dios". A nuestro parecer, Anselmo sostiene ambos tipos de argumentaciones. Y lo hace secuencialmente, como era propio en el medioevo, pues la fe no se daba sin racionalidad. Los argumentos del primer grupo pertenecen al Monologium, y los del segundo están representados por el "argumento ontológico" del Proslogium. En esta obra, la expresión "id quo maius cogitari nequit" hace referencia al "maius" por el cual la razón puede orientarse más allá de sí misma, con lo cual vemos que razón y fe van de la mano, pero que aquélla potencia a ésta.

En los tres primeros capítulos del Monologium hallamos las formas lógicas con que procede Anselmo. Dejaremos de lado el capítulo cuatro porque el argumento se halla en los tres primeros, y no tomaremos la secuencia de los capítulos dos y cuatro. Por abandonar el capítulo cuarto, sólo tomaremos del segundo lo que obedece al propósito de nuestro análisis y no a la demostración en extenso de Anselmo. No desconocemos que los especialistas discuten la existencia de dos, tres o cuatro argumentos, o de uno sólo en orden sucesivo a los capítulos uno-tres y dos-cuatro, o del capítulo uno al cuarto. Entre los que han estudiado estas secuencias podemos citar a Gilbert (1984), Vignaux (1947), Corti (1986). Sin embargo, a nuestro propósito, sólo 
importa el primer capítulo, puesto que argumenta la existencia de un bien absoluto, soberano e independiente, que es el fundamento por el cual las cosas que llamamos buenas son buenas. Veamos:

(...) todos los objetos entre los cuales existe una relación de más y menos, o de igualdad, son tales en virtud de una cosa que no es diferente, sino la misma en todos, sin que importe al caso el que éstas se hallen en ellos en proporción igual o desigual (Anselmo, 1952, p. 194).

Esta afirmación podría traducirse en la siguiente premisa: "todo objeto que presenta una cualidad, la posee en virtud de que esta cualidad se encuentra en alguna medida participada en él". Proposición que el texto anselmiano apuntala al señalar:"... por consiguiente, como es cierto que todas las cosas buenas, comparadas entre sí, lo son igual o desigualmente, es menester que sean buenas por algo que se concibe idéntico en todas" (Anselmo, 1952, p. 194); y unas líneas más adelante:"...pero como lo prueba incontestablemente la razón ya puesta en evidencia, es también necesario que todo lo que es... bueno, sea bueno por aquello precisamente por lo cual es bueno todo lo que lo es" (p. 196). Estas afirmaciones son formalmente equivalentes a: "y todo objeto bueno, es tal porque el bien participa en él". Así, Anselmo puede concluir: "...este bien es bueno por sí mismo, puesto que todo bien (toda bondad) viene por él. Síguese que todos los otros bienes proceden de otro que ellos, y que él sólo es por sí mismo" (1952, p. 196). Consecuencia irrecusable en "El bien presente en los objetos buenos, es el bien soberano que existe por-sí mismo". Ante todo ello debemos advertir un aspecto vivencial: la atención de Anselmo no está en la tautológica participación del bien, sino en el deseo de quien busca al bien en todo lo bueno. Y en el cumplimiento de dicho deseo, se halla al bien como absoluto y participado.

Esta argumentación es un modus ponendo ponens de la lógica sentencial. La misma estructura formal la hallamos en el capítulo segundo al sustituir "bondad" por "grandeza". Pero la sustitución no es banal, avanza a una profundización mayor de la razón impulsada por la fe. El argumento dice:

(...) del mismo modo hay que concluir necesariamente que hay un ser soberanamente grande, si se considera que todo lo que es grande lo es por un ser 
que es grande por sí mismo, grande, digo, no por la extensión, como un cuerpo, sino tal, que cuanto más grande es, mas digno y bueno es, como la sabiduría... es necesario que haya un ser a la vez soberanamente grande y soberanamente bueno, es decir, absolutamente superior a todo lo que existe (p. 196, cap. II).

Esta conclusión marca el antecedente de los capítulos dos a seis del Proslogium. Por lo pronto, observemos el capítulo tercero del Monologium, en donde muestra su ingenio argumentativo:

(...) porque todo lo que existe viene de algo o de la nada. Pero la nada no puede recibir el ser de la nada, porque ni siquiera se puede imaginar que haya algo sin causa; luego lo que existe no tiene el ser más que en virtud de otra cosa. Así las cosas, o la causa de lo que existe es única o hay varias; si hay varias, o convienen en un principio común que les ha dado el ser, o existen cada una de por sí, o se han creado mutuamente. Ahora bien, si provienen de un mismo principio, ya no tienen un origen múltiple, sino único. Si existen cada una por sí misma, hay que suponer la existencia de una fuerza o una naturaleza a la que es propio existir para sí, y de la que tienen su prerrogativa de existir por sí mismas; pero entonces es indudable que existen por aquel mismo y solo del cual tienen la propiedad de existir por sí mismos... en cuanto a una existencia por mutua comunicación, no hay ningún principio que permita admitirlo, porque sería contradictorio que una cosa recibiese el ser de aquella a la cual ella se lo da, y las relaciones mismas no se crean a sí mutuamente (p. 198, cap. II).

Lo anterior puede sintetizarse: "todo lo que existe, existe por algo o por nada. La segunda posición es absurda; así pues, todo lo que existe, existe por algo. Eso significa que todas las cosas existentes existen, o la una por la otra o por sí mismas o por una causa de existencia. Pero las dos primeras opciones son impensables: la primera porque es un absurdo recibir el ser precisamente de aquél a quien se le otorga; y la segunda por la contradicción de una pluralidad de causas incausadas, como lo sería una serie infinita consumada. Por tanto, sólo existe una sola causa incausada". Y así, la existencia de una causa incausada da seguridad al deseo de absoluto y rechaza la frustrante regresión al infinito. Llegar al "mayor" del cual no hay otro implica que dicho "mayor" es igual al summum. 
Anselmo concluye: "(...) solamente ella existe por sí misma, pero todo lo que existe por otro es menor que la causa que ha producido todos los seres y que existe por sí misma. Por lo cual, lo que existe por sí mismo es mayor que todo lo demás" (p. 200, cap. III). Esto le da pauta para el punto de partida del "argumento ontológico", pues el Monologium no sólo concluye la existencia de Dios, sino la atribución necesaria de ser soberanamente grande, lo más grande de todo. La unidad argumentativa entre las dos obras es indubitable y por tanto, en el caso de Anselmo, no se cumple la advertencia de Cabrera Villoro. Anselmo, lejos de dividir los argumentos de la existencia de Dios en los que parten del mundo y en los parten de la riqueza de Dios, los hila.

Ahora bien, los argumentos de esta primera índole tienen algunos presupuestos: considerar diversos grados de bondad o grandeza supone, como fenómeno aceptado, un modelo o "metro" que funciona como fundamento y norma de tales graduaciones. Por otra parte, la argumentación sólo puede aplicarse desde cualidades de los objetos que en sí mismas no impliquen una oquedad, imperfección o limitación. Es decir, esta inducción únicamente transita por la vía positiva. Además, si bien la solidez del argumento se encuentra en su consecuencia (como también veremos en el Proslogium y en Ockham), estas demostraciones poseen su fuerza en la intuición. La intuición anselmiana está cargada de emotividad; la ejemplaridad divina se intuye desde el corazón que desea las cualidades más grandes y más bondadosas en Dios. La persona de Dios, entonces, fungirá como modelo para cualquier cualidad en el mundo.

Dicha ejemplaridad es la clave para comprender el "argumento ontológico". Se cree, efectivamente, que las esencias o naturalezas de las criaturas se haIlan en la mente divina; éstas se conciben como ejemplos que constituyen el pensamiento absoluto. De este modo, Anselmo utiliza en el Proslogium la suposición, la consecuencia y la implicación material (aunque estas operaciones lógico-semánticas tardarán tres siglos más en aparecer con tales denominaciones), por ello puede decir:"...cuando me oye decir (refiriéndose al insensato) que hay un ser por encima del cual no se puede imaginar nada mayor, este mismo insensato comprende lo que digo; el pensamiento está en su inteligencia, aunque no crea que existe el objeto de este pensamiento" 
(Anselmo, 1952, p. 366, cap. II). Es decir, como dicho pensamiento "está en la inteligencia de quien lo piensa", al ser pensado, entonces necesariamente existe. Pero su realidad "pensada" se descubre en un esfuerzo de conciencia, no en el recorrido irreflexivo de una operación lógica irreversible y automática. La reflexión sobre el pensamiento no funciona sin el interés espiritual sobre lo que hay que pensar. El argumento de Anselmo exige la reflexión sobre la propia experiencia intelectual: pensar "lo mayor" en sí mismo lanza al espíritu al "más allá", pues por definición "lo mayor" siempre apunta más allá. No hay confusión de orden lógico y ontológico, porque la mente, al ver su orden lógico, se nota volcada hacia la realidad ontológica que mente y corazón desean.

Se requieren todavía otras precisiones: a) la inteligencia del insensato, como la de cualquier humano, no es absoluta. Por tanto, la existencia de sus pensamientos corresponde al orden de los entes de razón y no a los entes reales e independientes de quien los piensa; b) la sola comprehensión de un concepto no implica, dentro de las notas que lo constituyen, su extensión. Es decir, en términos de la lógica moderna; de la connotación no se sigue la denotación. Como ya lo había indicado Gaunilón, en la carta Pro-insipiente que le envía a Anselmo: no por pensar en una isla de oro, ésta existe; c) el sentido que posee la sentencia "este mismo insensato comprende lo que digo", no da pauta para considerar como real su referente. Este caso lo han ejemplificado varios semióticos de nuestro siglo (entre ellos Beuchot, 1979) aún con un toque de buen humor:"hoy por la mañana escalé una montaña de oro", y además lo hice "acompañado por el actual rey de la Argentina que es un tipo muy narigudo". Todos estos enunciados, si bien tienen sentido, no por ello poseen significación o referencia; d) no todo concepto (en su acepción de término mental, oral o escrito), por tomar un papel de representación, avala la existencia del representado. Este punto vincula a los términos y las sentencias bajo la teoría de la suppositio, ampliamente desarrollada por la semiótica medieval tardía en sus obras lógicas (Ockham, por ejemplo, desarrolla esta teoría en quince capítulos de la teoría de los términos de su Summa Logicae); e) Anselmo necesita agregar una estipulación al término mental para considerar la existencia necesariamente contenida en la comprehensión o connotación del concepto. Veamos el pasaje del Proslogium: 
(...) sin duda ninguna este objeto por encima del cual no se puede concebir nada mayor, no existe en la inteligencia solamente, porque si asífuera, se podría suponer, por lo menos, que existe también en la realidad, nueva condición que haría a un ser mayor que aquel que no tiene existencia más que en el puro y simple pensamiento. Por consiguiente, si este objeto por encima de lo cual no hay nada mayor estuviese solamente en la inteligencia, sería, sin embargo, tal, que habría algo por encima de él, conclusión que no sería legítima. Existe, por consiguiente, de un modo cierto, un ser por encima del cual no se puede imaginar nada, ni en el pensamiento ni en la realidad (Anselmo, 1952, p. 366).

El pensamiento moderno no duda de la existencia como una perfección. Pero de ahí a ser una perfección necesaria en Dios o en su descripción, tal y como hoy se interpreta a Anselmo, resultaría un juicio anacrónico. La lógica, necesariamente condicionada a la univocidad de los términos, no puede, per se, concluir la existencia de su objeto por condición de su comprehensión. La univocidad de la lógica impide ver su propio deseo y esfuerzo por alcanzar la preciada conclusión: Dios existe.

Esta miopía es padecida por algunos comentadores contemporáneos: no consideran a Dios un objeto susceptible de existir o no, porque si no existe necesariamente"no hay nada que su concepto pueda significar"3. A ellos parece pertinente replicar con las precisiones $\mathrm{c}$ ) y d) anotadas supra. Y además señalarles, que al suponer la existencia de Dios al modo de un concepto, no entienden nada del pensamiento de Anselmo; particularmente del maius, pues según la propia congruencia, nada habría por arriba de Él para juzgarlo. Él mismo no es un concepto: el maius no cabe como "uno apto para existir en muchos". Por tanto, efectivamente, "no hay nada que su concepto pueda significar", pues Dios, en cuanto maius, ni es concepto, ni con Él se busca significar algo. El espíritu anselmiano atiende mayormente al sentido referencial del maius que el de la comprehensión de "Dios"; por ello el texto desecha el término "Dios" y prefiere utilizar el maius. No toma el concepto ni adopta una descripción definida, sino que, una vez comprendido, hace entender que su sentido no se confina al entendimiento y, por tanto, también está en la realidad. Esto es, a juicio de Anselmo, lo peculiar de su prueba. Ahora bien,

3 Tal es el caso de Cabrera Villoro (1985, p. 381), quien sigue de cerca a Platinga y Hartshorne en su The ontological argument. 
equiparar mentalmente el maius a Dios es cuestión de creer en Dios como el mayor pensable, equivalencia menester de probar.

No vamos a revisar los ataques y defensas del trabajo anselmiano, pero debemos reiterar algunos puntos de la discusión que permiten observar el lugar que posee en la lógica medieval. Sus argumentos, metafísicos y teológicos, pertenecen a los dos tipos usuales: inductivo-semióticos y deducciones a partir de términos, definiciones o enunciados considerados axiomáticamente. Respecto a la semántica o contenido, reconocemos sin dificultad su raíz agustiniana; no así en su sintaxis. Anselmo aplicó a estos razonamientos las formas aristotélicas, añadiendo su innegable ingenio personal, tal como se manifiesta en las obras "semióticas". Ello explica la fuerza (que tanto ha dado para discutir) del "argumento ontológico" en el campo de las relaciones sintáctico-semánticas.

Gracias a lo que sabemos del tratado De Grammatico podemos vincular el Proslogium a la semiótica y el poder lógico del maius (Gilbert, 1993). Esta obra apunta la distinción entre significado y referencia. Beuchot lo ilustra de la siguiente manera: "La significación es el sentido o connotación, esto es la relación del término con la cosa como contenido conceptual o intensión, y no se relaciona con ella en cuanto cosa concreta, sino más bien se relacionan con ella en cuanto esencia" (1981, p. 42). Así, la significación es la cualidad de una substancia en tanto dicha cualidad corresponde a ser el significado de un signo. Para ejemplificar, Anselmo discute el término "gramático" que se dice adjetivamente de un hombre. Mientras que la referencia es la appelatio, es decir, "(...) es la referencia o denotación, esto es, la relación del término a una cosa existente y concreta" (Beuchot, 1981, p. 42). Ésta corresponde al objeto, a la substancia calificada con tal cualidad, como cuando decimos "Sócrates es gramático, puesto que sabe de gramática". Aquí la substancia es un apelativo. De este modo, los vocablos con significación pueden ser per se, puesto que significan directamente, como el caso de la cualidad, o podrán ser per aliud cuando lo hacen indirectamente como ocurre con la substancia, división que también está considerada en el texto de Anselmo. 
En el tratado De Veritate tenemos otra aplicación de lo anterior: en el capítulo segundo "De significationis veritate et de duabus veritatibus enuntiationis" se distingue, como el título lo hace notar, la diferencia entre significado y referencia respecto al enunciado y su verdad. Anselmo señala para el significado la naturaleza de la enunciación y su inmutabilidad; y para la referencia indica la rectitud y verdad de la misma considerándolas variables y accidentales. Nos dice a la letra:

(...) la rectitud y la verdad de la enunciación, expresando aquello para lo cual ha sido hecha, son, pues, distintas de aquellas que posee cuando significa que está hecha para significar. Estas son inmutables, aquéllas variables; éstas siempre la acompañan, porque forman parte de su naturaleza; aquéllas no siempre, sino de un modo accidental y según el empleo que de ella se quiera hacer (Anselmo, 1952, p. 496).

Con ello Anselmo muestra su dominio sobre las nociones de significación, sentido y referencia (aunque esta última, técnicamente no se encuentra en ninguno de sus textos), pues las distingue y utiliza en la solución de problemas escolares. Esto preludia la tardía teoría de la suppositio lógicosemántica que considerará la suplencia de los términos en tres aspectos: material, simple y personal. Pero la gran incógnita es si Anselmo conocía la distinción entre connotación y denotación, y si además utilizaba los diversos modos de suplantación, ¿por qué no dejó exento al "argumento ontológico" de los puntos vulnerables que son objeto de escarnio?, ¿el arzobispo de Canterbury no se percató del paso indebido que va de la posibilidad lógica a la actualidad real? Beuchot responde: "Anselmo, como platónico, concede cierta substancialidad a lo designado por los substantivos abstractos" (1981, p. 43). Por tanto, resultaría un realista exagerado; pero más bien de corte agustiniano/ejemplarista. Anselmo no es un teórico de la lógica, su preocupación y actitud es la de un creyente dinámico que orienta su fe en "lo mayor posible", incluso más allá del mismo pensamiento y no en lo que el pensamiento podría poseer en su inmanencia. Su contexto lo lleva a utilizar intuiciones e instrumentaciones semánticas y dialécticas para dar cabida a las creencias honestas de su corazón. 
Lógica (con este nombre) y semántica distintas encontramos en Guillermo de Ockham (1290, 1300-1349). Algunos estudiosos como Moody resaltan la importancia de Guillermo de Ockham "...cuya Summa Logicae, escrita sobre el 1326, inauguró el período de madurez de la lógica medieval" (1972, p. 82). Otros eruditos como Bochenski consideran que el "período de elaboración comienza aproximadamente con Guillermo de Ockham (1349/50) y dura hasta el fin de la Edad Media" (1967, p. 160). Sin embargo, otros especialistas como Stump (1989) lo consideran el cenit del desarrollo dialéctico medieval, tanto así que su habitual "nominalismo" es definido por De Andrés como un "proposicionalismo realista [el cual] constituye al ockhamismo en una filosofía del lenguaje" (1969, p. 236). Esta tesis se opone frontalmente a la opinión del experto okhamista Boehner (1958).

Lo cierto es que su "lógica" no es la más conocida del período (bastaría considerar las Summulae Logicales de Pedro Hispano), ni la más completa o elaborada (no tiene las ventajas cronológicas de la Logica Magna de Pablo de Venecia). Pero posee avances significativos y originales: completa la teoría de las segundas intenciones; aplica sistemáticamente la suppositio y la connotatio más allá de los signos arbitrarios, convencionales o artificiales, incluyendo conceptuales y naturales; declara a la suppositio en el campo exclusivo de la proposición. Según Copleston, uno de sus principales aportes es la teoría del signo: "[reconoce] la identidad de significación lógica que puede asignarse a palabras correspondientes en lenguajes distintos" (1979, p. 62, vol. 3). Asimismo, Lértora reconoce que Ockham aporta a la significación de los nombres infinitos la identidad como "todas las cosas finitas negativamente" (1987, p. 397). En este sentido, Ockham propone avances en la semiótica, en la teoría de la significación y en la lingüística.

Por otra parte, con los dos tratados de lógica posteriores a la Summa (el Compendium Logicae o Tractatus Logicae Minor y los Elementarium Logicae o Tractatus Logicae Medius), completa la instrumentación para atacar las entidades abstractas que habían multiplicado sin necesidad semiótica los tomistas y las excesivas formalidades de Escoto. Con esta semiótica, Ockham niega la "demostrabilidad" de la existencia de Dios; así lo expone en Quodlibetales (I, q. 1). La negación la ha mostrado ampliamente Webering (1955), con lo cual Ockham se ganó la fama de agnóstico. 
Ockham distingue entre "demostración" y "prueba". La primera la entiende como Aristóteles: un discurso científico que parte de principios o premisas evidentes racionalmente y que por ello son necesarias, y consecuentemente se establece el procedimiento silogístico. Para apreciar esto, tengamos en cuenta lo que señala Beuchot:

(...) todos los tipos de inferencia son considerados como partes y divisiones de la consecuencia... la importancia que tenía la consecuencia para los lógicos medievales difícilmente puede ponderarse. Constituía el contexto de todas las partes de la lógica, pues todos los tratados lógicos hacían referencia a la consequentia... que llega a ser la instancia superior de todas las operaciones lógicas (1981, p. 23).

De igual manera lo ilustra Moody:

(...) la más original e interesante de las contribuciones a la lógica acaecida en el período medieval, está fundada en la doctrina de la Consequentiae, la cual se desarrolló en la lógica del análisis proposicional, en la doctrina de la suppositio terminorum, que penetró a todos los tratados de lógica de los términos y también las funciones en el análisis semántico del significado, de la verdad y la denotación (1975, p. 377).

De manera que, si existiese un argumento cuyas premisas fueran tomadas de la revelación o alguna fuente fenomenológica-como los primeros casos de Anselmo-, dicho discurso no sería verdaderamente científico; cuando más, sería probatorio de premisas que no son necesarias. Ahora bien, según Ockham:"Al no ser la proposición Dios existe una proposición por sus propias notas, ni poder ser inferida de proposiciones inmediatamente evidentes, no cabe respecto de ella un auténtico proceso demostrativo" (Quodlibetales. I, q. 1.).

El argumento se centra en que la proposición Deus est no es un enunciado per se. En la noción de Dios no se encuentra contenido el predicado de la existencia. Ockham afirma algo en oposición contradictoria a Anselmo; sostiene que la connotación (comprehensión) del término "Dios" no implica, ni siquiera con una suppositio material, la denotación (extensión) de ser. Y tampoco el término "Dios" es connotativo (aquel que significa por modo de 
inherencia), según la propia acepción que le otorga Ockham en el capítulo 10 de la primera parte de la Summa.

Ante esta posición, la salida para recuperar el argumento de Anselmo sería una inferencia material. Sin embargo, largas y eruditas son las discusiones sobre el conocimiento de Ockham acerca de la implicación material como una especie de la consecuencia material, en virtud de las características de los términos dentro de las proposiciones. Con respecto a lo anterior, puede verse la polémica entre Boehner (1958) y Moody (1953) versus Mullick (1971) y Adams (1971). De cualquier forma, al advertir que las consecuencias materiales se dividen en "simples" y "sólo por ahora", hacemos referencia a las simples. Moody (1976, pp. 90-91) ha explicado que las consecuencias de esta clase satisfacen la fórmula [en ningún caso ocurre que a la vez $p$ y no $q$ ]; pero valen en virtud de la "materia" o contenido de los términos descriptivos utilizados en los enunciados que componen el argumento. Su definición en una tabla veritativa reza: el antecedente no puede ser en tiempo alguno verdadero y el consecuente, falso. Ockham da los siguientes ejemplos: "si un hombre corre, existe Dios. El hombre es un asno, luego Dios no existe" (Ockham, Summa Logicae, cap. III, citado en Bochenski, 1967, p. 205).

Nos asombran estos ejemplos, pues para el consecuente "existe Dios" podemos enunciar materialmente un antecedente siempre verdadero, y formalmente un antecedente indistinto, siempre y cuando no posean conexión esencial, ni causal con el consecuente. Es decir, en sentido estricto no hay consecuencia. Pero sintácticamente, en cualquier caso, Dios existe: pues así como una proposición imposible implica cualquiera otra, de igual modo una proposición necesaria es implicada por cualquiera.

Pero ¿por qué no aceptar el "argumento ontológico"?, ¿por qué oponerse tan frontalmente a Anselmo?4. Nuestra hipótesis afirma que para Ockham es imposible una implicación o consecuencia material que fundamente el enunciado "Dios existe". Desde la teoría de la suppositio, con su división y jerarquía, es impensable una proposición así.

4 Advirtamos que no existen referencias donde Ockham critique los argumentos anselmianos. No hemos hallado un texto así, ni creemos que exista. Sencillamente hacemos la comparación y sólo podríamos contextualizarla en disputa entre realistas exagerados y nominalistas con respecto a los universales. Pero concedemos que el término "Dios" de ningún modo representa un concepto universal. 
La suposición material del término "Dios", dado que haría alusión a los vocablos y grafemas, es viable en la Summa Logicae:

Hay suposición material cuando el término no supone significativamente, sino supone o por su voz o por su escrito. Como es patente aquí, "hombre es nombre" supone por sí mismo, y sin embargo, no significa a sí mismo. Similarmente es esta proposición:"hombre se escribe", puede haber suposición material, porque el término supone por aquello que es escrito (cap. 64).

Cuando decimos "Dios existe", con suposición material, nos referimos a la palabra "Dios" que, en efecto, existe. Pues existe una palabra tal, compuesta de cuatro letras. Pero no es sobre el término "Dios" del que trata la intención teológica del argumento. Por otra parte, la suposición simple no se ejerce como signo de las cosas; sino como un reemplazo de otros signos lingüísticos en la Summa Logicae: "Hay suposición simple cuando el término supone por la intención del alma, pero no se toma significativamente" (cap. 64). Situación que no ocurre en las premisas ni en la conclusión del argumento de Anselmo. Para el de Canterbury ni Dios, ni lo grande, ni lo bueno son especies, ni signos, ni segundas intenciones meta-lingüísticas.

La suposición personal es la más importante y la verdaderamente utilizada en la semiótica. Según Ockham, consiste en:

La suposición personal, universalmente, es aquélla que se da cuando el término supone por su significado, sea cuando aquel significado es una cosa fuera del alma, o un escrito, o cualquier otra cosa imaginable. Así que en cualquier momento en que el sujeto o el predicado de la proposición suponga por su significado, es decir, que se considere significativamente, siempre hay suposición personal (Summa Logicae, cap. 64).

Por tanto, se ejerce como signo de las cosas, ocupando su puesto y reemplazando en la proposición a todas y cada una de las entidades singulares. Así, el término "Dios" resulta inapropiado también para la suposición simple, pues ella supone la existencia sólo de las realidades singulares y sus respectivos nombres universalizados en la mente. Las entidades singulares, en tanto tales, no son susceptibles de demostración necesaria y universa; y ninguna de estas dos alternativas corresponde a Dios, si se desea una demostración teológica. 
Ockham saca a Dios del universo de las demostraciones y lo recluye al de las pruebas. Su prueba no está fincada en la necesidad de las "premisas" o "antecedentes", tampoco en la evidencia universal de su punto de partida, y ni siquiera en su verdad. Ockham rechaza las conocidas pruebas escolásticas sobre la existencia de Dios en los Quodlibetales, en los Commentarium in Sententiis y en la Expositio Super Physicam. Ahí examina la vía del movimiento, de la causalidad eficiente y de la finalidad; las desecha porque sus premisas proceden de la fe. En tanto principios son inadmisibles por carecer de evidencia o porque presentan un processus in infinitum; sin embargo, los reconoce como argumentos "probables", pues la existencia de un primer motor o fin natural es más probable que su inexistencia. Son más probables porque son más razonables que una serie infinita actual. Además, agrega otros candidatos razonables para un primer motor:ángeles u otros inferiores a Dios; así como para una causa primera: cuerpos celestes. En ello se percibe la influencia teológica y astronómica de las concepciones aristotélico-ptolomeicas en la Edad Media.

Ockham propone una prueba similar a la causalidad eficiente para la existencia de Dios: prueba de la conservación de los entes contingentes. Procede de la conservación hasta un Conservador de manera paralela a la vía que transita desde lo creado hasta un Creador. Dice en Philosophia Naturales:

Digo que por eficiente, según lo que la cosa dice, inmediatamente no puede percibirse, no puede probarse el principio eficiente; es por eficiente que lo que la cosa dice continúa en la existencia, esto puede ser bien probado por la conservación... Pero la conservación no es por un proceso al infinito porque alguna esencia infinita lo actué, lo cual es imposible; ya que todo lo conservante de lo otro, ya sea mediato, ya sea inmediato, es al mismo tiempo conservado; y por esa razón todo lo conservante requiere actualizar todo lo conservado. Tan no es así que los productos requieren ser actualizados por los productores, mediata o inmediatamente. Por esta razón, aunque pudiera ponerse a los productos en un proceso al infinito sin actualizarlos infinitamente, no se puede poner también lo conservado en un proceso al infinito sin actualidad infinita (q. 136).

La probabilidad de la existencia divina está en la sucesión de pensamientos más razonables que en los argumentos contrarios. Pero el criterio de lo "más 
razonable o más probable" no puede resolverse al interior de la lógica, ni de la semiótica. ¿Por qué la vía de la conservación y no la producción?, ¿por qué es "más probable" una sucesión que otra? Los criterios pertenecen a lo meta-lingüístico. La lógica tiene necesidad de recurrir a este nivel.

Algunas operaciones lógicas como la simple aprehensión, la abstracción o la inducción, son en sí mismas insuficientes. Requieren de un conocimiento pre-científico, base de la ciencia y de mayor fundamentación. Aristóteles lo reconoce al definir este saber como epagogé (Anal. Post., 1881 a. 40, Ética Nicomáquea, 1139 b. 28), noción que seguramente aprendió de la platónica anagogé. Asentemos que Ockham niega a la abstracción y la intuición en el plano natural (potentia ordinata) la fuerza para acceder al conocimiento de Dios; no así en el plano sobre-natural (potentia absoluta), pues dice en Commentarium in Sententiis:

Toda inteligibilidad puede captar semejantes conocimientos. Por lo tanto, así como una criatura puede conocer intuitiva y abstractivamente, así también Dios... Acerca del segundo artículo digo que la potencia absoluta de Dios es poder de tal naturaleza que aumenta el conocimiento, así que al mismo tiempo sea intuitivo y además abstractivo. Sin embargo es difícil probar esto, no obstante puede ser persuadido... (l, prol,1.).

La intuición abstractiva-inductiva funda las operaciones lógicas y funciona para acceder a Dios y elaborar conceptos y términos universales. Procede elaborando síntesis desde las cualidades particulares, reconociendo un común denominador en la multiplicidad. Así, los predicados de perfecciones son atribuidos en Dios. Y el concepto de éste sigue en el marco anselmiano: el más excelente del cual no puede pensarse nada mayormente excelente. Vignaux (1948, pp. 46-47) señala la tensión latente en Ockham: conoce a Dios intuyendo el concepto común de los entes (el ser). Le resulta exclusivo, pero no procede con algún tipo de analogía. No es nuevo entonces el tema de la "diferencia".

La intuición es el proceso meta-lógico, o más bien, ante-lógico que funda los criterios de lo evidente, lo razonable y lo aceptable. Ya en otros sitios hemos tratado esto (Aguirre, 1988). Ahora, a propósito de Ockham, recordamos las 
palabras de Rábade:“...todo conocimiento abstractivo de un objeto supone previamente la intuición del mismo" (1966, p. 153). Copleston también señala que"...nos es imposible tener por nuestra potencia natural un conocimiento abstractivo de algo en sí mismo sin un conocimiento intuitivo de esa cosa" (1979, p. 91, vol. 3). Y en referencia a la Philosophia Naturalis (cap. Il, fol 2, col 2) de Ockham, Larre y Bolzán dicen: “.. . la naturaleza misma de la física le impide hablar de una omnivalente posibilidad de demostración, rehabilitándose consiguientemente el valor del conocimiento intuitivo" (1988, p. 74).

Anselmo y Ockham coinciden en la intuición de la realidad supra sin término. El primero, como realista exagerado, prácticamente incluye el predicado"existencia" en el sujeto “Dios". Mientras el último, en el nominalismo, se opone a tal inclusión al negar los conceptos denotativos. Hace alusión a la intuición de los particulares para elevarse al fundamento, por encima incluso de los universales. Y a pesar de las diferencias ante el problema de los universales, y de sus distintas semióticas, ambos aplican la instrumentación raciocinativa en auxilio de la fe y la teología.

\section{Referencias}

Adams, M. (1971). Did Ockham know of Material and Stric Implication?. EnNotre Dame Journal of Formal Logic, (12).

Aguirre, J. (1988). Sobre la hipótesis. En Revista de Filosofía UIA, (62).

Aguirre, J. (1990). Conocimiento metódico y no metódico. En Revista de Filosofía UIA, (68).

Anselmo. (1952). Obras completas. Madrid: B.A.C.

Beuchot, M. (1979). Elementos de semiótica. México: U.N.A M.

Beuchot, M. (1981). La filosofía del lenguaje en la Edad Media. México: U.N.A.M.

Bochenski, I. M. (1967). Historia de la Lógica Formal. Madrid: Gredos. 
Boehner, P. (1958). Does Ockham know of Material Implicatio? En Collected Articles on Ockham. Nueva York: St. Bonaventure Franciscan Institute.

Cabrera, M. (1985). Sobre el argumento ontológico; historia de su defensa. En Revista de Filosofía U.I.A., (54).

Colish, M. L. (1983). St Anselm's Philosophy of Language. En Anselm Studies. London: Kraus International.

Copleston, F. (1979). Historia de la Filosofía (vol. 3). Barcelona: Ariel.

Corti, E. (1986). El bien y la esencia. En Stromata, (XLII).

Corti, E. (1994). La prueba anselmiana y el código ontológico. En Cuadernos del Sur, (XXV).

De Andrés, T. (1969). El nominalismo de Guillermo de Ockham como filosofía del lenguaje. Madrid: Gredos.

Evana, G. R. (1975). The 'Recrue Technician'. Varieties os Paradoxes in The Writings of St. Anselm. En Vivarium.

Evana, G. R. (1979a). St. Anselm's Definitions. En Archivum Latinitatis Medii Aevi.

Evana, G. R. (1979b). St. Anselm's Technicals Terms of Grammar. En Latomus.

Gilbert, P. (1984). Dire l'ineffable. París: Beauchesne.

Gilbert, P. (1993). Significatio nel De grammatico e maius nel Proslogium di S. Anselmo. En Studia Patavina, (40).

Gombocz, W. \& Dazeley, H. L. (1979). Interpreting Anselm as Logician. En Synthese

Hartman, R. S. (1960). Prolegomena to a Meta-anselmian Axiomatic. En Review of Metaphysics.

Henry, D. P. (1967). The Logic of Saint Anselm. Oxford: Clarendon Press. 
Henry, D. P. (1970). Saint Anselm as Logician. En Sola ration. Stuttgart: F. Frommann Verlag.

Larre, O. \& Bolzán, J. (1988). El tema epistemológico en Ockham. En Revista de Filosofía UIA, (61).

Lértora, C. A. (1987). Los nombres infinitos según Ockham. En: Revista de Filosofía U.I.A., (60).

Moody, E. A. (1953). Truth and consequencia in Medieval Logic. Nueva York: North- Holland.

Moody, E. A. (1975). Studies in medieval Philosophy, Science, and Logic. Berkeley: University of California Press.

Moody, E. A. (1976). Lógica medieval europea. En Prior, A.N. Historia de la Lógica. Madrid:Técnos.

Mullick, M. (1971). Does Ockham accept Material Implicatión? En Notre Dame Journal of Formal Logic, (12).

Ockham, G. (1958). Commentarium in Sententiis, ed. por Boehner. En Collected Articles on Ockham. Nueva York:The Franciscan Institute St. Bonaventure.

Ockham, G. (1958). Philosophia Naturales, (ed. por Boehner). En Collected Articles on Ockham. Nueva York:The Franciscan Institute St. Bonaventure.

Ockham, G. (1958). Quodlibetales (ed. por Boehner). En Collected Articles on Ockham. Nueva York: The Franciscan Institute St.Bonaventure.

Ockham, G. (1958). Summa Logicae (ed. por Boehner). En Collecte Articles on Ockham. Nueva York: The Franciscan Institute St. Bonaventure.

Rábade, S. (1966). Guillermo de Ockham y la Filosofía del siglo XIV. Madrid: CSIC.

Riviere, J. (1937). Saint Anselm logician. En Revue des Sciences Religieuses.

Stump, E. (1989). Dialectic and its place in the development of medieval logic. Nueva York: Cornell University Press. 
Vignaux, P. (1947). Structure et sens du Monologion. En Revue des Sciences Philosophiques et Théologiques, (XXXI).

Vignaux, P. (1980). Nécessité des Raisons dans le Monologion. En Revue des Sciences Philosophiques et Théologiques, (LXIV).

Vignaux, P. (1981). Nominalisme au XIV siècle. París: Vrin-Reprises.

Webering, D. (1953). Theory of demostration according to William Ockham. Nueva York: St. Bonaventure.

Wilbanks, J. J. (1973). Some (Logical) Trouble for Anselm. En New Scholasticism. 